\title{
Seropositive Reaction Rates of 9 B-Cell Epitopes of the SARS-CoV-2 Spike Protein and the Relationship between the Epitopes and Neutralizing Antibody
}

\author{
Li Zhu $^{a, b}$ Yunwen Zhang ${ }^{c}$ Zhengrong Yang ${ }^{b}$ Baisheng Li $^{d}$ Tiejian Feng ${ }^{b}$ \\ Xuan Zou ${ }^{b}$ Jianfan $\mathrm{He}^{\mathrm{b}}$ Taiping He ${ }^{\mathrm{e}}$ Junling $\mathrm{Li}^{\mathrm{e}} \mathrm{Ning} \mathrm{Liu}^{\mathrm{b}}{\mathrm{Wei} \mathrm{Li}^{\mathrm{a}}}^{\mathrm{a}}$ \\ Xiaohui Wang ${ }^{b}$ \\ aSchool of Laboratory Medicine and Life Sciences, Wenzhou Medical University, Wenzhou, China; bShenzhen Center \\ for Disease Control and Prevention, Shenzhen, China; 'West China School of Public Health and West China Fourth \\ Hospital, Sichuan University, Chengdu, China; ' $G u a n g d o n g$ Provincial Center for Disease Control and Prevention, \\ Guangzhou, China; 'School of Public Health, Guangdong Medical University, Zhanjiang, China
}

\section{Keywords}

Severe acute respiratory syndrome coronavirus $2 \cdot$ Spike protein · Epitopes · Neutralizing antibody

\begin{abstract}
Objective: The aim of the study was to analyze the relationship between serum antibody and neutralizing antibody titers in convalescent coronavirus disease 2019 (COVID-19) patients with different disease severities, and the seropositive reaction rates of 9 reported B-cell epitopes of severe acute respiratory syndrome coronavirus 2 (SARS-CoV-2). Methods: Serum IgG and total antibody titers of 165 convalescent COVID-19 patients were determined by chemiluminescence, the serum neutralization antibody titers were determined by microneutralization assay, and the $\mathrm{S} / \mathrm{CO}$ values of 9 peptides were detected by indirect enzyme-linked immunosorbent assay. Correlations between the aforementioned indexes were statistically analyzed, and differences in patients with different diseases severities were evaluated. Results: IgG, total antibody, and neutralizing antibody titers
\end{abstract}

karger@karger.com www.karger.com/int

Karger $\stackrel{\text { ' }}{5}$

GOPEN ACCESS
(C) 2021 The Author(s)

Published by S. Karger AG, Basel

This is an Open Access article licensed under the Creative Commons Attribution-NonCommercial-4.0 International License (CC BY-NC) (http://www.karger.com/Services/OpenAccessLicense), applicable to the online version of the article only. Usage and distribution for commercial purposes requires written permission. increased with disease severity. The positive rate of the receptor-binding region (RBD) was $100 \%$, and the average positive rate for all the 9 peptides was above $50 \%$ in 165 patients. IDf showed the highest rate of positivity (86.06\%), with a rate of $95 \%$ for the (IDf + IDa) pattern. Moreover, S/CO values of RBD and mix (IDh) were significantly correlated with IgG, total antibody titers, and neutralizing antibody titers $(p<0.001)$, whereas the $\mathrm{S} / \mathrm{CO}$ values for other 8 peptides showed no obvious correlation. Conclusion: In this study, a large sample was used to confirm that the peptide IDf had a high positive reaction rate for all patients $(86.06 \%)$ and also had the highest detection rate in asymptomatic patients (86.67\%). Only long peptide and mixed peptide showed correlation with neutralizing antibody titers, suggesting that the ability of SARS-CoV-2 antibody to neutralize virus infectivity may require the interaction of multiple sites.

(c) 2021 The Author(s).

Published by S. Karger AG, Basel

Li Zhu, Yunwen Zhang, Zhengrong Yang, and Baisheng Li contributed equally to this work.
Correspondence to:

Wei Li, liwei@wmu.edu.cn

Xiaohui Wang, wangxh@szcdc.net 


\section{Introduction}

The epidemic of severe acute respiratory syndrome coronavirus 2 (SARS-CoV-2) posed a serious threat to the global public health $[1,2]$. Scientists have carried out valuable research on this topic, and the study of B-cell epitopes is of great significance for the development of vaccines and antibody detection reagents. The spike (S) protein of SARS-CoV-2 plays an important role in the process of virus binding to the angiotensin-converting enzyme 2 receptor and cell membrane fusion as well as entry into host cells. Thus, blocking the binding of S protein and angiotensin-converting enzyme 2 receptor is an effective strategy to prevent coronavirus entering target cells [3-5]. On July 1, 2020, Zhang et al. [6] identified 9 linear immunodominant (ID) epitopes by using the serum samples from 39 patients with coronavirus disease 2019 (COVID-19) and found that 4 linear immune loci were located in the receptor-binding region (RBD). In the present study, we used serum samples from $165 \mathrm{CO}$ VID-19 patients to confirm the positive reaction rates of 9 epitopes and analyzed the relationship between the S/ $\mathrm{CO}$ values of 9 epitopes, the IgG concentration, and neutralizing antibody titers.

\section{Materials and Methods}

\section{Serum Source of Discharged Patients}

Blood samples and clinical information of 165 discharged COVID-19 patients were collected during March 5, 2020 to May 12, 2020, in Shenzhen, China. These discharged patients were treated at the Third People's Hospital of Shenzhen and met the COVID-19 discharge criteria. According to the classification of clinical symptoms in the Novel Coronavirus Pneumonia Diagnosis and Treatment Plan (Provisional 7th Edition) promulgated by the National Health Commission of the People's Republic of China [7], 165 COVID-19 patients were divided into asymptomatic patients (30, $18.18 \%)$, mild patients $(22,13.33 \%)$, moderate patients (106, $64.24 \%$ ), and severe and critically ill patients $(7,4.24 \%)$ (there was one critical case included to facilitate the statistics). The median age of all patients was 33 years, and the proportion of female patients was $52.72 \%$. At the same time, serum samples from 20 healthy people were collected as the negative control group. The control population was free of SARS-CoV-2 infection, and HIV, hepatitis $\mathrm{C}$, and syphilis serum tests were all negative. To ensure biosafety, the serum samples were incubated at $56^{\circ} \mathrm{C}$ for $30 \mathrm{~min}$ to avoid potential risks. This study was reviewed by the Ethics Committee of Shenzhen Center for Disease Control and Prevention in Guangdong Province (QS2020070048).

\section{Total Antibody and IgG Antibody Detection}

The Caris200 Automatic Chemiluminescence Instrument and Chemiluminescence Kit (Xiamen Wantai Biological Pharmacy Enterprise Co., Ltd, Xiamen, China) were used to detect IgG and total antibody levels. Both the instrument and reagent were certified by the China National Medical Products Administration (NMPA) (National Instrument Registration Certificate 20203400198). This method used the SARS-CoV-2 S-RBD as the detection antigen, and the detection indicator was COI value. The COI was the sample detection value/cutoff value, and the cutoff value was the average serum test result from 5 healthy people. The serum samples with COI above 1 were considered positive.

\section{Microneutralization Assay}

Neutralizing antibody titers were measured by microneutralization assay. The SARS-CoV-2 virus isolate (20SF014/Vero-E6/3) and the Vero-E6 cell line were used for this assay. Vero-E6 cells were seeded in 96-well plates at $1 \sim 2 \times 10^{4}$ cells $/ 100 \mu \mathrm{L}$ and cultured for $12 \mathrm{~h}\left(37^{\circ} \mathrm{C}, 5 \% \mathrm{CO}_{2}\right)$. After heat inactivation at $56^{\circ} \mathrm{C}$ for $30 \mathrm{~min}$, the serum was diluted by gradient dilution of $1: 4,1: 16,1: 64,1: 256$, and 1:1,024. The SARS-CoV-2 virus was incubated with serum at a titer of $104.67 \mathrm{TCID}_{50} / 50 \mu \mathrm{L}$ for $2 \mathrm{~h}$. Next, $100 \mu \mathrm{L}$ of diluted serum and virus sample mixture were added to wells containing the Vero-E6 cells. Viral positive controls were prepared with the titers of $100 \mathrm{TCID}_{50} / 50 \mu \mathrm{L}, 10 \mathrm{TCID}_{50} / 50 \mu \mathrm{L}, 1 \mathrm{TCID}_{50} / 50 \mu \mathrm{L}$, and 0.1 $\mathrm{TCID}_{50} / 50 \mu \mathrm{L}$, respectively, and cultured with the samples simultaneously. When complete cytopathic effect occurred in the positive control wells of $\mathrm{TCID}_{50} / 50 \mu \mathrm{L}$ virus, the level of cytopathogenic effect for each well was recorded. For each serum sample, the reciprocal of the highest dilution of serum that could protect $50 \%$ of the cell well from cytopathic effect was defined as the SARSCoV-2 neutralizing antibody titer of that sample. The titer was above 1:4, which was considered positive. All operations are performed in a level 3 biosafety laboratory.

\section{Peptide Synthesis and Dissolution}

The peptides used in this study were synthesized by Sangon Biotech Co., Ltd. (Shanghai) and transported in the form of freezedried powder. The quality of all peptides was detected by mass spectrometry, and the purity was $98 \%$. Each peptide segment was 20-25 amino acid residues in length, with 5 overlapping amino acid residues in adjacent sequences. The solvent used for the dissolution of the peptide was prepared according to the instruction manual, that is, and dissolved in ultra-pure water or $75 \%$ ethanol, $25 \% \mathrm{ddH}_{2} \mathrm{O}$ (double-distilled water) and 5\% formic acid.

\section{Indirect Enzyme-Linked Immunosorbent Assay}

The peptide solution with final concentration of peptides $(2.5$ $\mu \mathrm{g} / \mathrm{mL}$ ) was prepared (except peptide IDe: $0.5 \mu \mathrm{g} / \mathrm{mL}$ ) by dissolving in carbonate buffer. The S-RBD (CUSABIO; \# CSB-MP3324 GMY1b1) was used as the positive antigen control, with a final concentration of $0.2 \mu \mathrm{g} / \mathrm{mL}$. Each well of a 96-well plate (\# 42592; Corning) was added with $100 \mu \mathrm{L}$ of peptide solution and coated overnight at $4^{\circ} \mathrm{C}$. The plate was washed 5 times with 300 $\mu \mathrm{L}$ wash solution $(0.05 \%$ Tween- 20 and $1 \%$ bovine serum albumin in PBS). Blocking with $300 \mu \mathrm{L}$ blocking solution (5\% skim milk powder and $0.1 \%$ Tween-20 in PBS) for overnight at $4^{\circ} \mathrm{C}$. The plate was washed 5 times and added $100 \mu \mathrm{L} /$ well of inactivated serum from patients diluted at 1:80 in blocking buffer, followed by incubation at $37^{\circ} \mathrm{C}$ for $1 \mathrm{~h}$ after shaking. Each sample was test duplicate. The plate was washed and added $100 \mu \mathrm{L} /$ well of solution containing rabbit anti-human IgG HRP conjugate (\# 6759, diluted at 1:130,000; Abcam), followed by incubation at $37^{\circ} \mathrm{C}$ for $1 \mathrm{~h}$. The plate was washed and added $100 \mu \mathrm{L} /$ well of TMB (\# EK0011; Multi
30

Intervirology 2022;65:29-36

DOI: $10.1159 / 000517717$
Zhu/Zhang/Yang/Li/Feng/Zou/He/He/Li/ Liu/Li/Wang 


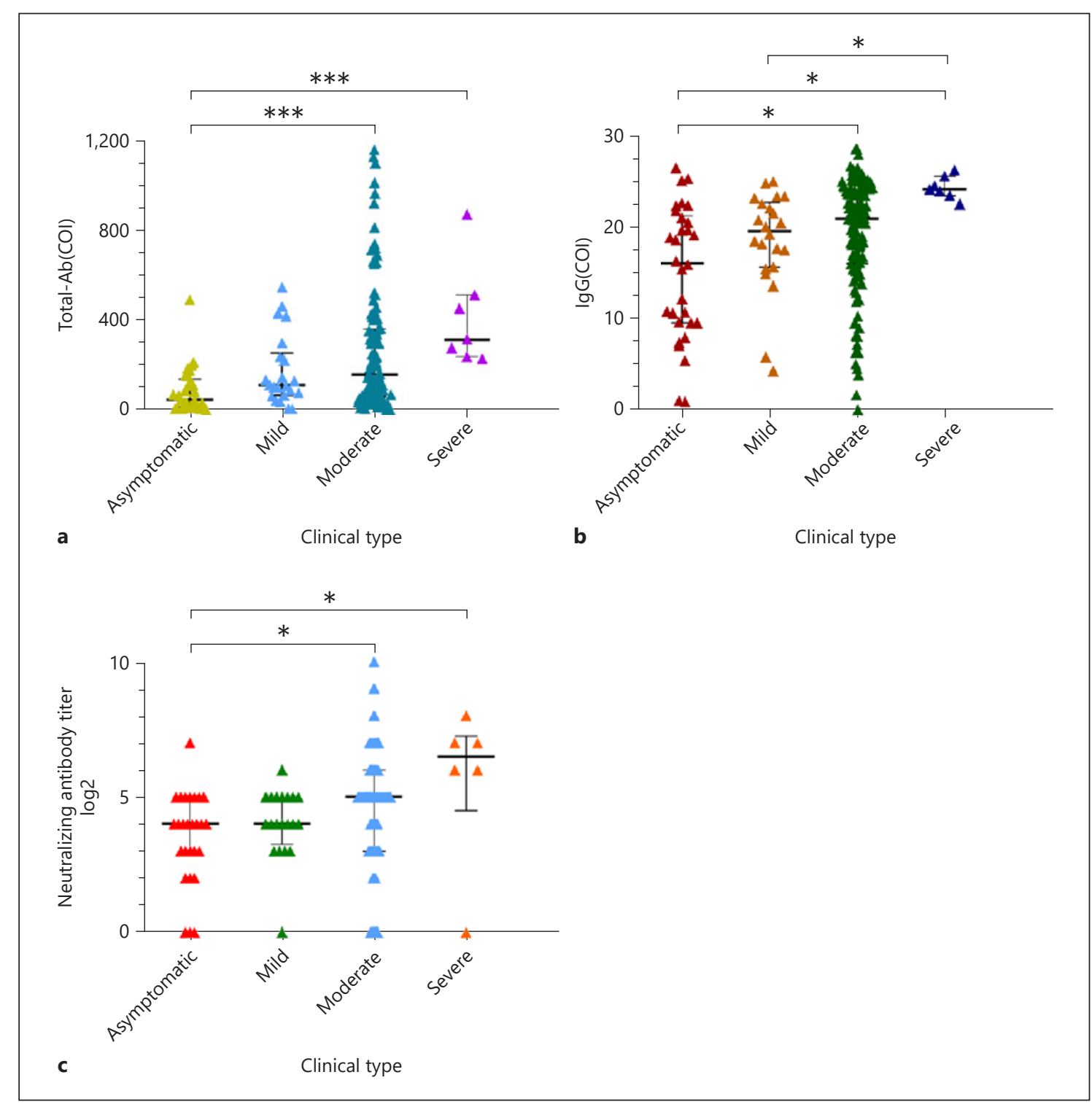

Fig. 1. Distribution of serum antibody levels in 165 convalescent COVID-19 patients with different disease severity. Total antibody levels (COI) (a), IgG levels (COI) (b), and neutralizing antibody titers (c). COI: sample detection value/cutoff value. Each small triangle represented the detection value of 1 sample. Data are shown as median (IQR). IQR, interquartile range; COVID-19, coronavirus disease 2019.

Science). After shaking, plates were incubated at room temperature in the dark for $25 \mathrm{~min}$ and added $100 \mu \mathrm{L} /$ well of stop solution. The OD value was determined at $450 \mathrm{~nm}$ as the maximum absorption wavelength and $630 \mathrm{~nm}$ as the reference wavelength. Samples from a healthy person were used as negative controls, and 6 negative control wells were set for each plate. The cutoff value was based on the mean value of negative controls plus 3 times the standard deviation. The serum samples with an S/CO above 1 were considered positive.

B-Cell Epitopes of the SARS-CoV-2 Spike Protein

\section{Data Analysis}

SPSS Statistics 21, GraphPad Prism 8.0, and Origin 2021 were used for statistical analysis and plotting. The $\chi^{2}$ test was used to analyze the difference in the positive rate of different epitopes. The Kruskal-Wallis $H$ test was used to analyze the difference in antibody titers (COI) and epitope S/CO values. Correlation analysis was carried out by using Spearman's rank correlation, and the $3 \mathrm{D}$ structure of protein was analyzed online using by NCBI website. When $p<0.05$, the difference was considered statistically significant: ${ }^{*} p<0.05,{ }^{* *} p<0.01$, and ${ }^{* * *} p<0.001$. 

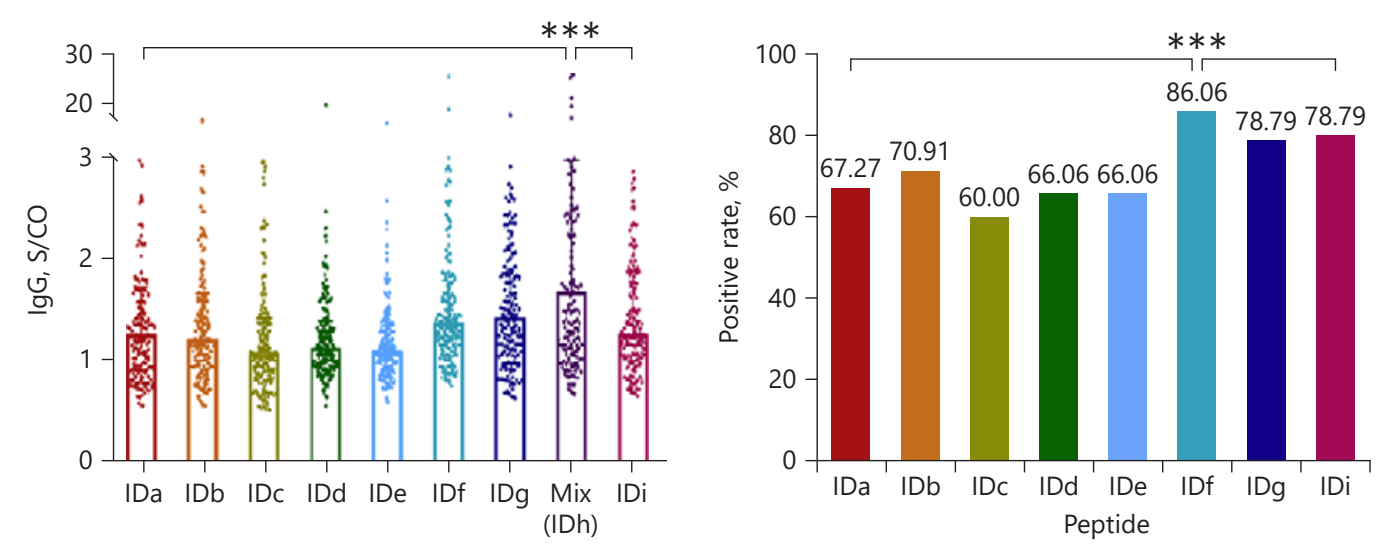

a

Peptide

b
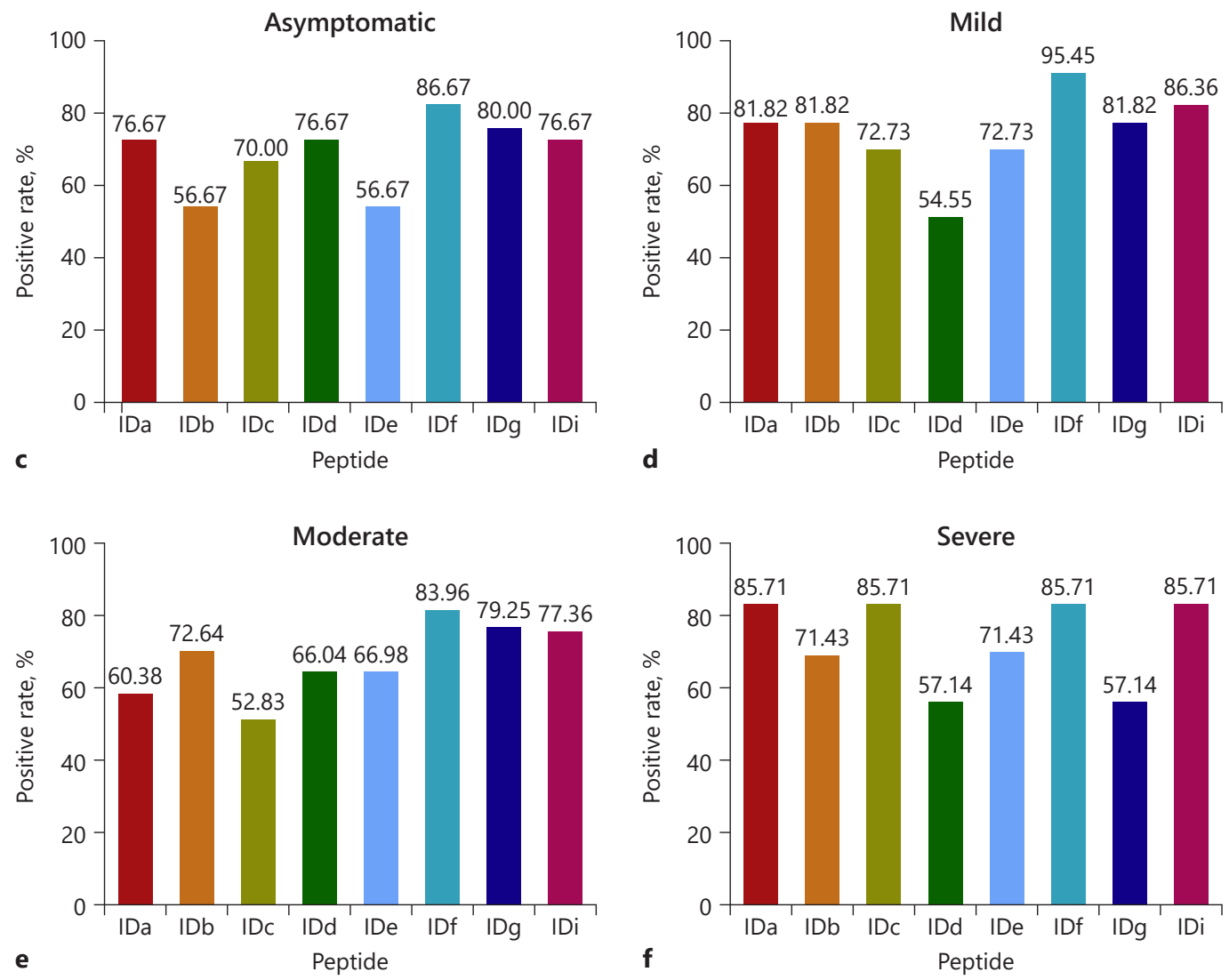

Fig. 2. S/CO values of each peptide and the positive reaction rates of the peptides in convalescent serum from COVID-19 patients by using indirect ELISA. a The S/CO values of each peptide in 165 convalescent COVID-19 serum samples. Each point represented per patient, and the data are represented as the median (interquartile range). $\mathbf{b}$ The overall seropositive reaction rates of the 8 short peptides in 165 convalescent COVID-19 serum samples. c-f The seropositive reaction rates of 8 short peptides in 165 convalescence COVID-19 patients with different disease severity. S/CO value: sample OD value/cutoff value, the sample with S/CO > 1 was regarded as positive, and the cutoff value was the average OD value of healthy people plus 3 times the SD. COVID-19, coronavirus disease 2019; ELISA, enzyme-linked immunosorbent assay. 


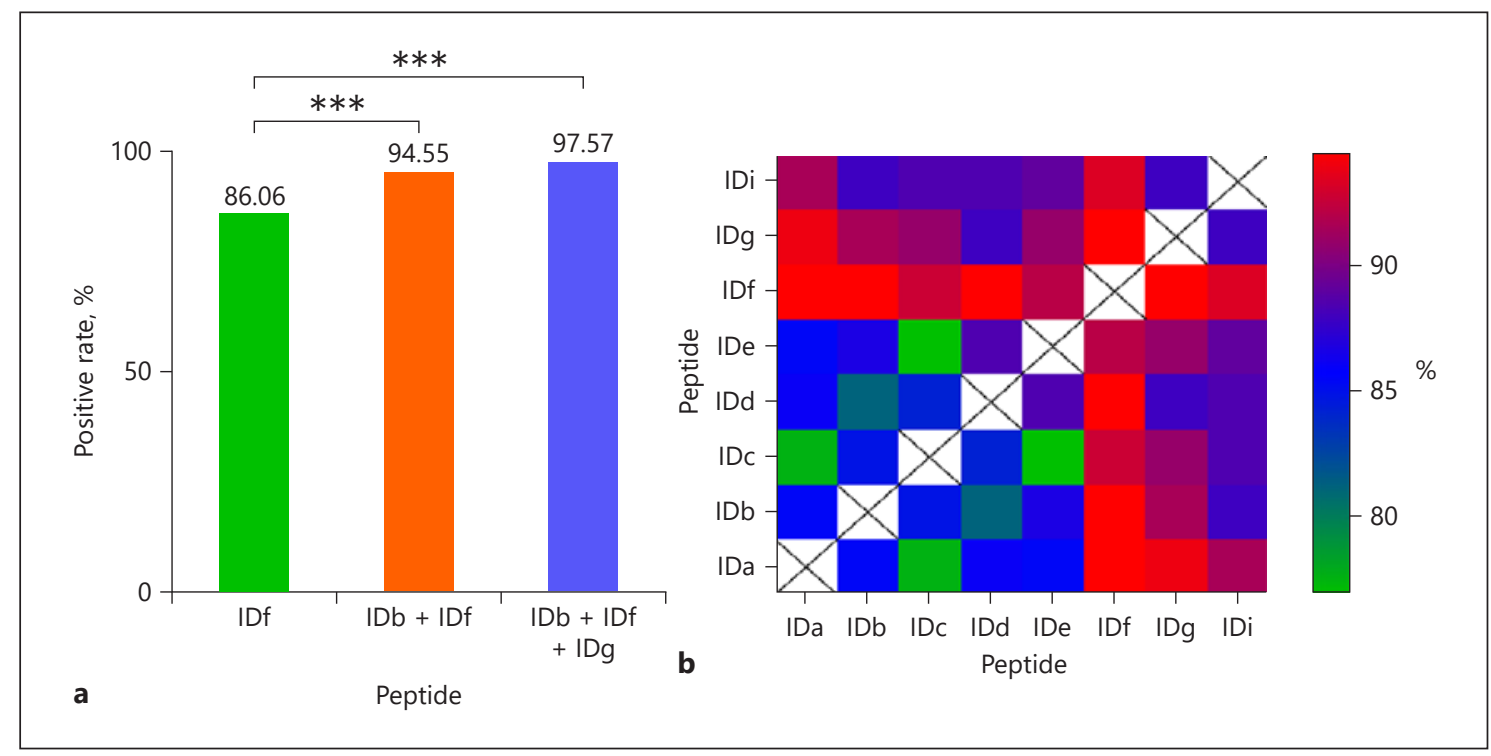

Fig. 3. Seropositive reaction rates of different peptide combinations. a The positive rates of IDf combination with different peptides. For combinations of 2 and 3 peptides, (IDf + IDb) and (IDf + IDb + IDg) showed the highest positive reaction rate, respectively. $\mathbf{b}$ The positive rate of any pairwise combination of the 8 peptides was used to analyze in serum samples from 165 COVID-19 patients. In the figure, red indicates a high positive rate, and green indicates a low positive rate. The positive rate of a combination was the cumulative positive rate of 2 or 3 peptides for 165 serum samples. COVID-19, coronavirus disease 2019.

\section{Results}

Description of the Antibody Levels of the Samples

This study measured total antibody, IgG antibody, and neutralizing antibody titers in 165 convalescent COVID-19 patients. The positive rates of total antibody and IgG were $98.78 \%$ (163/165). The median COI (interquartile range $[\mathrm{IQR}])$ of total antibody and IgG were 128.39 (267.52) and 20.43 (8.39), respectively. The neutralizing antibody detection rate was $90.67 \%(136 / 150)$, and the median (IQR) was 1:32 (1:24). Overall, IgG, total antibody, and neutralizing antibody titers increased with disease severity (shown in Fig. 1a-c).

\section{Positive Rates of the Reactions between Peptides and Serum}

According to enzyme-linked immunosorbent assay results, the median S/CO (OD value/cutoff value) of the reactions between peptides (IDa to IDi) and serum ranged from 1.07 to 1.68 peptide mixture ( $\mathrm{mix}$ [IDh]) among the 165 convalescent patients. The S/CO value of mix (IDh) was higher than that of other peptides $(p<0.001)$ (shown in Fig. 2a). Mix (IDh) is a mixed long peptide fragment with amino acid sites ranging from 522 to 646, including
125 amino acids. In terms of detection rate, we were unable to compare it with other short peptides.

As shown in figure, the overall positive reaction rates of 8 short peptides were as follows: (1) RBD was used as the positive control the positivity rate was revealed as $100 \%$ when reacting with all the serum samples from patients, with a median S/CO (IQR) of 18.46 (10.87). (2) For the short peptides, the overall positive reaction rates were $67.27 \%$ for IDa, $70.91 \%$ for IDb, $60 \%$ for IDc, $66.06 \%$ for IDd, $66.06 \%$ for IDe, $86.06 \%$ for IDf, $78.79 \%$ for IDg, and $78.79 \%$ for IDi. The positive rates of all peptides were $>50 \%$ (shown in Fig. 2b). (3) Among these 8 peptides, the positive rate of IDf was significantly higher than that of the other peptides $(p<0.001)$. Regarding samples of different clinical types, IDf also showed an advantage in detection, especially in asymptomatic and mild patients, reaching a rate of $>95 \%$ (shown in Fig. 2c-f). (4) Analysis of different peptide combinations showed that the overall positive rate was $94.55 \%$ for (IDb + IDf), and the positive rate of (IDb + IDf + IDg) was $97.57 \%$ (shown in Fig. 3a), both of which were significantly higher than the detection rate of IDf $(p<0.001)$. In addition, the combination of IDf with any peptide showed a positive reaction rate $>90 \%$ (shown in Fig. 3b). 


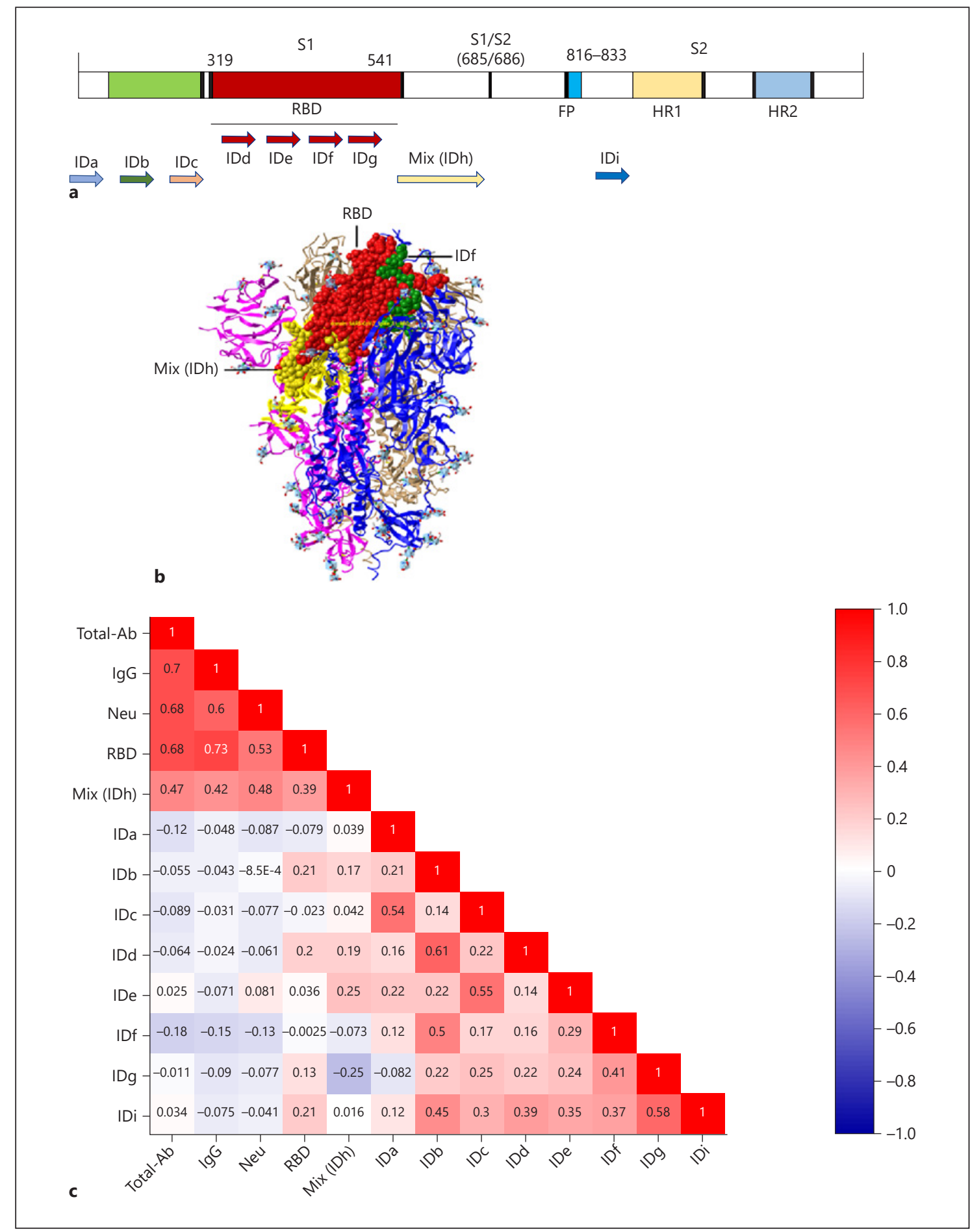

Fig. 4. Location of each peptide in the S protein genome and S protein 3D structure. And correlation analysis between peptide S/CO values with antibody levels. a Representative structures of some functional domains of the SARS-CoV-2 S protein. RBD, receptorbinding domain; FP, fusion peptide; HR1, heptad repeat region 1; HR2, heptad repeat region 2. b Spatial locations of the mix (IDh), IDf, and RBD peptides on the 3D structure of the SARS-CoV-2 S protein. The $3 \mathrm{D}$ structure of the SARS-CoV-2 S protein was ob- tained from the NCBI website (PDB ID: 6VXX). The 3 peptides are represented by 3 different colors: red denotes the RBD, yellow denotes $\operatorname{mix}$ (IDh), and green denotes IDf. c Correlation relationship between $\mathrm{S} / \mathrm{CO}$ values of peptides with antibody titer. The number in the figure was the Spearman correlation coefficient, and the darker the color meant the stronger the correlation. SARS-CoV-2, severe acute respiratory syndrome coronavirus 2 ; RBD, receptorbinding region. 


\section{Correlation Analysis between Peptides and Antibody}

Levels

The S protein of SARS-CoV-2 had 2 subunits: S1 and S2 [8-10]. Among the 9 peptides used in this study, IDi was located on the S2 subunit, whereas the others are located on S1 and 4 peptides, IDd, IDe, IDf, and IDg, are located in the RBD (shown in Fig. 4a). From the perspective of spatial location, mix (IDh) was close proximity to the RBD, and IDf is located in the RBD (shown in Fig. 4b).

The analysis showed that the S/CO values of RBD showed the highest correlation with IgG levels $(r=0.73$, $p<0.001)$. Conversely, the S/CO values of all short peptides exhibited no correlation with IgG levels or total antibody levels. In the analysis of the correlations between the neutralizing antibody titers and peptides, the correlation coefficients between the neutralizing antibody titers and $\mathrm{S} / \mathrm{CO}$ values of the RBD and $\mathrm{mix}$ (IDh) were 0.53 and 0.48 , respectively $(p<0.001)$. In addition, correlation coefficients of mix (IDh) with total antibody levels, IgG levels, and RBD levels were $0.47,0.42$, and 0.39 , respectively $(p<0.001)$ (shown in Fig. 4c), while there was no correlation between neutralizing antibody titers and the other peptides, including the 4 located in the RBD (shown in Fig. 4c).

\section{Discussion}

In July 2020, Zhang et al. [6] reported 9 B-cell linear epitopes of the SARS-CoV-2 S antigen. S antigen is the main antigen for virus neutralizing antibodies. Nowadays, research on its antigenic epitopes has important significance for vaccine development and diagnosis. At the beginning of this study, few $S$ antigen epitopes have been reported, and the sample sizes of those studies were relatively small. The 9 peptides used in this study are the latest B-cell linear epitopes published by Zhang et al. [6].

Zhang et al. [6] reported the positive reaction rates of the 9 peptides to 35 serum samples were above $50 \%$. In the present study, the 9 peptides were individually validated via serum samples from 165 convalescent COVID-19 patients. The results showed positive reaction rates of 9 peptides ranging from $60 \%$ to $86.06 \%$, with the rate of short peptides IDf being significantly higher than that of other peptides, even higher than mix (IDh). Moreover, IDf displayed a significant detection advantage when compared with the other 3 peptides located in the $\mathrm{RBD}$ region. These results indicated that IDf was a dominant B-cell epitope, which was of great significance for the development of SARS-CoV-2 detection kits and may

B-Cell Epitopes of the SARS-CoV-2 Spike Protein have reference value for the development of peptide vaccines.

Amrun et al. [11] found that both the sensitivity and specificity of S14P5 (TESNKKFLPFQQFGRDIA) for the detection of serum samples from 79 COVID-19 patients were above $86 \%$, higher than that of the 9 peptides in this study. These results further indicated that the function of each B-cell epitope is diverse, and it is valuable to screen for dominant epitope. In this study, mix (IDh) was a mixture of 6 peptides that contained the S14P5 amino acid sequence; however, its positive reaction rate of $83 \%$ may due to the following reasons: (1) the amino acid sequence of S14P5 was distributed on 2 different peptides in mix (IDh) (shown in online suppl. Table 1; see www.karger. com/doi/10.1159/000517717 for all online suppl. material), affecting its binding ability (2) or the different sample size and the composition ratio of patients with different disease severity may have caused the difference. Among 165 patients in this study, 19.4\% (32) were asymptomatic; asymptomatic patients usually had lower antibody titers.

Antibodies were the main evaluation indicator of vaccine efficacy, while neutralizing antibody titers could effectively reflect the blocking of antibodies against viruses. This study found that the positive rates of IgG and total antibody in 165 convalescent COVID-19 patients were both $98.8 \%$, consistent with previous reports [12-14]. The positive reaction rate of the neutralizing antibody in the 150 tested patients was $90.67 \%$, and the median (IQR) was 1:32 (1:24). However, the S/CO values of the 8 peptides were not correlated with the neutralizing antibody titers. Notably, IDd, IDe, IDf, and IDg were located in $\mathrm{RBD}$, and none of these 4 epitopes was correlated with neutralizing antibody. The result indicated that not all the B-cell epitopes located in the RBD was associated with neutralization capacity. However, this study showed that the RBD and mix (IDh) were significantly correlated with the neutralizing antibody titers, suggesting that the ability of novel coronavirus antibody for virus neutralization may require multiple epitope sites; however, the existence of predominant epitopes cannot be ruled out.

This study conducted comprehensive antibody detection and analysis of serum samples from 165 convalescent COVID-19 patients, but the number of epitopes we analyzed were limited. With the continuous discovery of new B-cell epitopes of SARS-CoV-2, the peptide combinations for serological detection are constantly improving [15-17]. This study found a high overall positive reaction rate and a high detection rate for IDf in asymptomatic patients. The combination of the IDf with other peptides 
showed obvious detection advantages in improving the positive rate of serological detection, which is of great significance for the detection of antibodies against COVID-19.

\section{Statement of Ethics}

This research comply with the guidelines for human studies, and the research was reviewed by the Ethics Committee of Shenzhen Center for Disease Control and Prevention in Guangdong Province (QS2020070048), which agreed that the subjects were exempt from signing the informed consent. The supporting materials were attached.

\section{Funding Sources}

This work was supported by the Shenzhen Committee of Scientific and Technical Innovation grant (JCYJ20180508152244835), Sanming Project of Medicine in Shenzhen (SZSM202011008) and Shenzhen Key Medical Discipline Construction Fund (SZXK064).

\section{Author Contributions}

W.L. and X.W. conceived and designed the research. L.Z., W.Y., J.L., and T.H. performed experiments, and acquired and analyzed the data. L.Z., W.L., X.W., Z.Y., B.L., T.F., X.Z., J.H., N.L., and J.L. wrote the manuscript. All the authors read and critically reviewed the manuscript.

\section{Data Availability Statement}

Data are made available upon reasonable request to the author.

\section{References}

1 Wang C, Horby PW, Hayden FG, Gao GF. A novel coronavirus outbreak of global health concern. Lancet. 2020;395(10223):470-3.

2 Chan JF, Yuan S, Kok KH, To KK, Chu H, Yang J, et al. A familial cluster of pneumonia associated with the 2019 novel coronavirus indicating person-to-person transmission: a study of a family cluster. Lancet. 2020; 395(10223):514-23.

3 Walls AC, Park YJ, Tortorici MA, Wall A, McGuire AT, Veesler D. Structure, function, and antigenicity of the SARS-CoV-2 spike glycoprotein. Cell. 2020;181(2):281-92.e6.

4 Letko M, Marzi A, Munster V. Functional assessment of cell entry and receptor usage for SARS-CoV-2 and other lineage B betacoronaviruses. Nat Microbiol. 2020;5(4):562-9.

5 Hoffmann M, Kleine-Weber H, Schroeder S, Krüger N, Herrler T, Erichsen S, et al. SARSCoV-2 cell entry depends on ACE2 and TMPRSS2 and is blocked by a clinically proven protease inhibitor. Cell. 2020;181(2):271-80. e8.

6 Zhang BZ, Hu YF, Chen LL, Yau T, Tong YG, $\mathrm{Hu}$ JC, et al. Mining of epitopes on spike pro- tein of SARS-CoV-2 from COVID-19 patients. Cell Res. 2020;30(8):702-4.

7 Wei P-F. Diagnosis and treatment protocol for novel coronavirus pneumonia (trial version 7). Chin Med J. 2020;133(9):1087-95.

8 Yi Z, Ling Y, Zhang X, Chen J, Hu K, Wang $\mathrm{Y}$, et al. Functional mapping of B-cell linear epitopes of SARS-CoV-2 in COVID-19 convalescent population. Emerg Microbes Infect. 2020;9(1):1988-96

9 Jiang S, Du L, Shi Z. An emerging coronavirus causing pneumonia outbreak in Wuhan, China: calling for developing therapeutic and prophylactic strategies. Emerg Microbes Infect. 2020;9(1):275-7.

10 Yao H, Song Y, Chen Y, Wu N, Xu J, Sun C, et al. Molecular architecture of the SARS CoV-2 virus. Cell. 2020;183(3):730-38.e13.

11 Amrun SN, Lee CY, Lee B, Fong SW, Young BE, Chee RS, et al. Linear B-cell epitopes in the spike and nucleocapsid proteins as markers of SARS-CoV-2 exposure and disease severity. EBioMedicine. 2020;58:102911.

12 Lou B, Li TD, Zheng SF, Su YY, Li ZY, Liu W, et al. Serology characteristics of SARS-CoV-2 infection after exposure and post-symptom onset. Eur Respir J. 2020;56(2):2000763.

13 Zhao J, Yuan Q, Wang H, Liu W, Liao X, Su $\mathrm{Y}$, et al. Antibody responses to SARS-CoV-2 in patients with novel coronavirus disease 2019. Clin Infect Dis. 2020;71(16):2027-34.

14 Lassaunière R, Frische A, Harboe ZB, Nielsen ACY, Fomsgaard A, Krogfelt KA, et al. Evaluation of nine commercial SARS-CoV-2 immunoassays. medRxiv. 2020;2020.04.09. 20056325.

15 Wang C, Gao Z, Shen K, Cao J, Shen Z, Jiang K, et al. Safety and efficiency of endoscopic resection versus laparoscopic resection in gastric gastrointestinal stromal tumours: a systematic review and meta-analysis. Eur J Surg Oncol. 2020;46(4 Pt A): $667-74$.

16 Phan T. Genetic diversity and evolution of SARS-CoV-2. Infection, genetics and evolution. Infect Genet Evol. 2020;81:104260.

17 Poh CM, Carissimo G, Wang B, Amrun SN, Lee CY, Chee RS, et al. Two linear epitopes on the SARS-CoV-2 spike protein that elicit neutralising antibodies in COVID-19 patients. Nat Commun. 2020;11(1):2806. 